\title{
Prevalence of hepatitis $C$ virus infection in potentially malignant disorders and oral squamous cell carcinoma-an immunohistochemical study
}

\begin{abstract}
Objectives: To evaluate the expression of antibody Hepatitis C Virus Non Structural Protein 3 (HCVNS3) in potentially malignant disorders (PMDs) and oral squamous cell carcinoma (OSCC) by using immunohistochemistry and to correlate it with 5 years survival rate in patients with OSCC.
\end{abstract}

Materials and Method: The study group consisted of 50 histopathologically diagnosed cases of PMDs and 100 histopathologically diagnosed cases of OSCC with a known 5 year survival status. The paraffin embedded tissue section of $4 \mu \mathrm{m}$ was obtained from archival tissues which were then stained for routine Hematoxylin and Eosin and the immunohistochemistry was carried out using the monoclonal antibody HCVNS3. The squamous cells whose cytoplasm stained brown were considered positive for the expression.

Results: Out of 50 PMDs, 3 showed positive expression for anti HCVNS3 and out of 100 OSCC, 7 showed positive expression for the antibody. Among 100 OSCC, 49 were alive and 51 were dead at 5 year after the surgical treatment

Conclusion: HCV could be an etiologic agent in the development of PMDs and OSCC. The co-infection with HCV in OSCC is indicative of poor survival in patients.
Volume 2 Issue 6 - 2018

\author{
Rajul Ranka,' Minal Chaudhary, ${ }^{2}$ Swati Patil,'2 \\ Madhuri Gawande, ${ }^{2}$ Anuj Jain, ${ }^{3}$ Preethi \\ Sharma², Shree Bajaj ${ }^{2}$ \\ 'Department of Oral Pathology and Microbiology, Maharashtra \\ University of Health Sciences, India \\ ${ }^{2}$ Department of Oral Pathology and Microbiology, Datta Meghe \\ Institute of Medical Sciences, India \\ ${ }^{3}$ Department of Trauma and Emergency Medicine, All India \\ Institute of Medical Sciences, India
}

\begin{abstract}
Correspondence: Rajul Ranka, Assistant Professor, Department of Oral Pathology and Microbiology, Maharashtra Institute of Dental Sciences and Research, MIT Campus, Latur, Maharashtra, India, Tel +9l 855496949l, Email rajul.ramka@gmail.com
\end{abstract}

Received: May 13, 2017| Published: December 18, 2018

Keywords: hepatitis C virus, hyperkeratosis, oral lichen planus, oral squamous cell carcinoma, oral submucous fibrosis

\begin{abstract}
Abbreviations: $\mathrm{HCC}$, hepatocellular carcinoma; HCV, hepatitis $\mathrm{C}$ virus; HCVNS3, hepatitis C virus non structural protein 3; HLA, human leukocyte antigen; IHC, immunohistochemistry; JNK, c Jun $\mathrm{N}$ - terminal kinase; MAP, kinase- mitogen activated protein kinase; NF-kB, nuclear factor kappa B; OLP, oral lichen planus; OSCC, oral squamous cell carcinoma; OSMF, oral submucous fibrosis; PCR, polymerase chain reaction; PI3K, Phosphoinositide-3-kinase; PMD, potentially malignant disorder; TGF- $\beta$, transforming growth factor beta; Wnt, wingless $t$
\end{abstract}

\section{Introduction}

Oral squamous cell carcinoma (OSCC) is the sixth most common cancer worldwide. ${ }^{1}$ OSCC is characterized by a cancerous tissue growth of the epithelial cells of the oral cavity and is brought about by an array of genomic abnormalities which may have developed over a period of time. ${ }^{2}$

The identified risk factors of oral cavity cancer include tobacco use, smoking, alcohol consumption, race, chewing of betel leaves and areca nuts, low socioeconomic status and genetic susceptibility. ${ }^{3}$ Also, various bacteria's, fungi and viruses have an important role in oncogenesis. Still there is no established etiology for OSCC and many new etiological agents are forthcoming. These new causative agents have to be evaluated for their role in etiology of OSCC.

Since last few decades researchers are taking enormous interest in the role that viruses play in the development of oral cancer. Approximately $12 \%$ of human cancers worldwide are caused by oncovirus infection, with more than $80 \%$ of cases occurring in the developing world. ${ }^{4} \mathrm{HCV}$ has been classified as the first member of a genus called Hepacivirus within the family Flaviviridae. Hepatitis C Virus (HCV) is a significant public health problem. Approximately $3 \%$ of the world's population has constant HCV infection. ${ }^{5}$ In addition to the association with hepatocellular carcinoma; HCV infection has been associated with other neoplasms including smoking and alcoholrelated cancers, such as cancers of the pancreas, lung, kidney, and the oropharygeal cancer and non-Hodgkin lymphoma. ${ }^{6}$

HCV RNA has been found in salivary tissue suggesting that $\mathrm{HCV}$ is a sialotropic virus. Continual exposure of this virus to oral mucosa can lead to genetic instability in cells. ${ }^{7}$ Long term infection from HCV may lead to an uncustomary immunologic response. The virus is thought to replicate in oral mucosa and attract $\mathrm{HCV}$ specific $\mathrm{T}$ lymphocytes, and thus may have role in pathogenesis of oral mucosal lesions. ${ }^{8}$ No scientific study has been carried out in India in relation to incidence of $\mathrm{HCV}$ and its co-existence with potentially malignant disorders (PMDs) and OSCC to date.

Thus, the present study aims at addressing this need and at assessing the expression of anti HCVNS3 (Hepatitis C Virus Nonstructural protein 3) protein in normal mucosa, PMDs and OSCC.

\section{Materials and methods}

The present study design was a retrospective randomized controlled trial study carried out in the Department of Oral Pathology and Microbiology, Sharad Pawar Dental College and Hospital, Sawangi (Meghe), Wardha, after receiving consent from the patients and approval from Institutional Ethical Committee, Datta Meghe Institute of Medical Sciences, Sawangi (M), Wardha, Maharashtra, India. 
The study included 50 histopathologically diagnosed cases of PMDs and 100 histopathologically diagnosed cases of OSCC of various grades from the departmental archives. 50 samples of normal oral mucosa served as control for the study.

The paraffin embedded tissue section of $4 \mu \mathrm{m}$ was obtained from archival tissues which were then stained for routine Hematoxylin and Eosin and the immunohistochemistry was carried out using the primary monoclonal antibody Hepatitis C Virus NS3 [V/1859] 100 ug (GeneTex).

\section{Procedure}

Slides were pre-coated with 3-amino propyltriethoxy silane for section adhesion and to minimize section loss due to pre-treatment. The sections obtained were subjected to the following:

-Hematoxylin and Eosin (H \& E) stain.

-Immunohistochemistry staining by HCVNS3 antibody.

Steps in immunohistochemical staining-

Sections were hydrated with increased grades of alcohol and brought to distilled water and treated with hydrogen peroxide $\left(\mathrm{H}_{2} \mathrm{O}_{2}\right)$ to eliminate endogenous peroxidase activity. Then antigen retrieval with tri-sodium citrate was carried out. The tissue was incubated sequentially with-

A. Primary antibody HCVNS3.

B. DAKO Envision system HRP labelled polymer detection system.

C. DAB (3, 3 Diamino benzidine substrate solution) - This results in formation of a colored precipitate at the tissue antigen sites.

Visualization was aided by counter staining with hematoxylin.

Negative control: In every batch of IHC staining, one paraffin section was stained where the primary antibody was omitted. This served as a negative control.

Assessment of immunohistochemical stained sections with anti HCVNS3:

The sections stained were examined under Leica DMLB2 (Leica Microscope) at 40X. Anti HCVNS3 immunoreactivity was confined primarily to the cytoplasm. One hundred cells from 5 randomly selected representative fields under high power $(\times 400)$ of each section were counted. All the epithelial cells whose cytoplasm stained dark brown were considered positive for the staining (Figure 1).

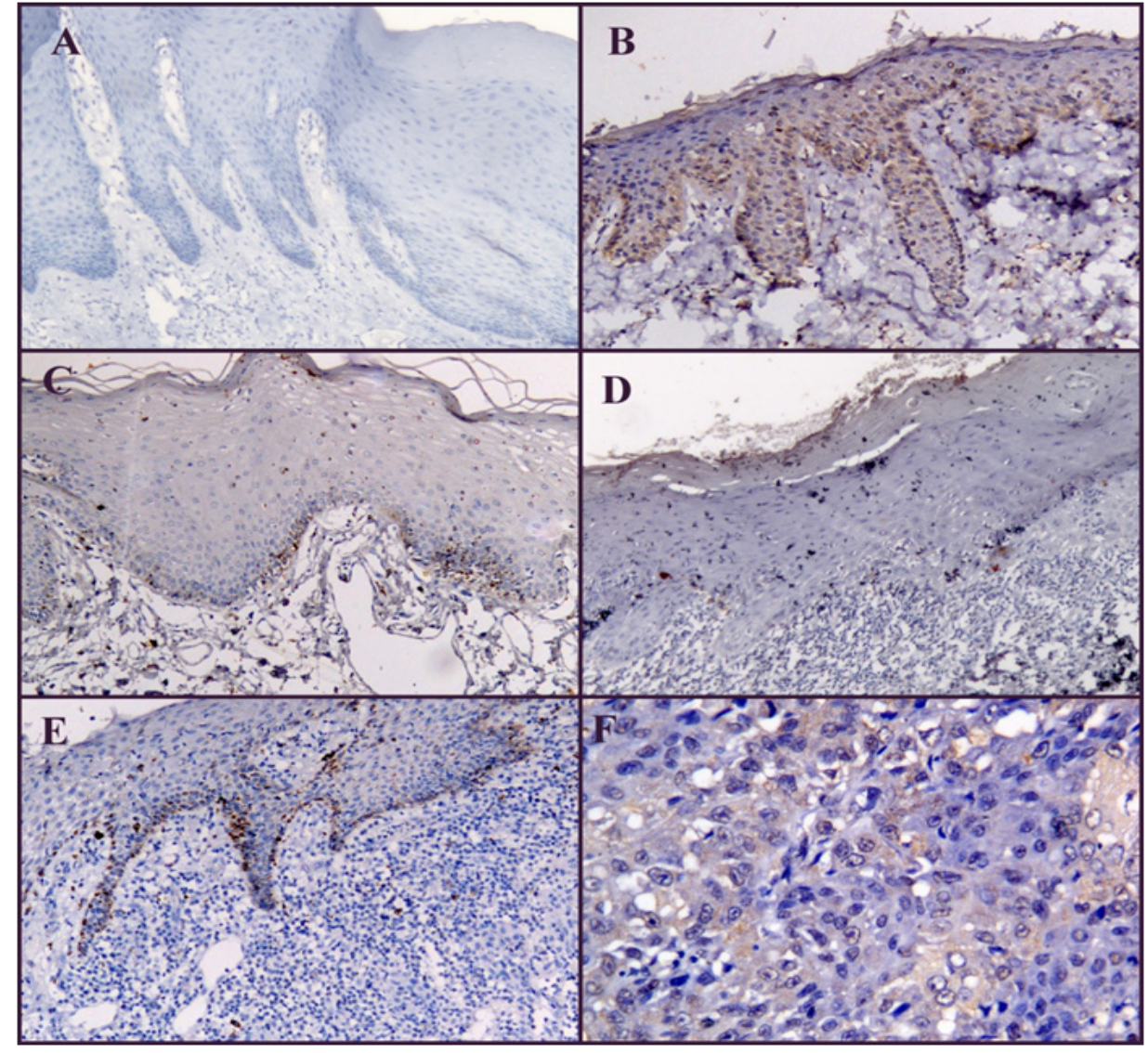

Figure I (A) Negative expression of HCVNS3 in normal mucosa (I0x); (B) Positive expression of HCVNS3 in oral submucous fibrosis (I0x); (C) Positive expression of HCVNS3 in Hyperkeratosis (I0x); (D) Negative expression of HCVNS3 in oral lichen planus (I0x); (E) Positive expression of HCVNS3 in epithelium of oral squamous cell carcinoma (I0x); (F) Positive expression of HCVNS3 in neoplastic epithelial cells of squamous cell carcinoma (40x). 


\section{Results and discussion}

Out of 50 PMDs, 3 showed positive expression for anti HCVNS3 while 47 showed negative expression. Out of 100 patients with OSCC, 7 patients showed positive expression for anti HCVNS3 and 93 patients showed negative expression for the antibody (Table 1).

The expression of anti HCVNS3 was evaluated based on the cells exhibiting cytoplasmic staining. The expression of anti HCVNS3 was seen as brown granular staining in the cytoplasm of basal cell layer of epithelium and in invaded neoplastic epithelial cells. Out of 50 normal mucosa samples only one showed positive expression. Among 50 PMDs, 3(6\%) showed positive expression for anti HCVNS3. Among which, out of 32 oral submucous fibrosis patients $2(6 \%)$ were positive, among 10 hyperkeratosis $1(10 \%)$ was positive for anti HCVNS3 and among 8 oral lichen planus (OLP) $0 \%$ were positive for anti HCVNS3. This is in contradiction of the data by Lodi et al. ${ }^{9}$ Nagao Y et al. ${ }^{10}$ and Nagao Y \& Tanaka J et al. ${ }^{11}$ who stated that one of the most frequently reported oral extrahepatic manifestations of $\mathrm{HCV}$ infection is lichen planus. Also, in a Brazilian cross-sectional study, there was a significant association between oral lichen planus and hepatitis C. ${ }^{12}$ Petti $\mathrm{S}$ et al. ${ }^{13}$ in their meta-analysis and case control study stated that although HCV and OLP were significantly associated, the majority of OLP patients were not affected with HCV.
By our study we could not show any correlation of OLP with HCV in Indian population, though further studies are required to validate this data. This could also be due to the small sample size. A study conducted by Jaber MA et al. ${ }^{14}$ in British population stated that there is no association between $\mathrm{HCV}$ infection and oral epithelial dysplasia. Analysis of our data shows slightly increased incidence of HCV with OSMF and hyperkeratosis. Further studies with more number of patients are needed to substantiate this data.

Among 100 samples of OSCC, $7 \%$ showed positive expression for anti HCVNS3. This is the first study conducted in Indian population. The reported prevalence of $\mathrm{HCV}$ infection in Indian population is below $2 \%$ and in Maharashtra it is $0.09 \%$ as studied by Mukhopadhya $\mathrm{A}^{14}$ Therefore an obvious increase in HCV incidence in OSCC patients is noted. So, this study points to a positive correlation between HCV and OSCC, though further studies in this regard are required taking larger population into consideration.

In the present study males were more frequently affected with both PMDs and OSCC as compared to females. Potentially Malignant Disorders were more prevalent in 3 rd decade with a mean of 39 years while OSCC was more prevalent in $6^{\text {th }}$ decade of life with a mean of 53 years. The buccal mucosa was the most prevalent site in both PMDs and OSCC (Table $2 \& 3$ ).

Table I Expression of anti HCVNS3 in potentially malignant disorders (PMDs) and oral squamous cell carcinoma (OSCC)

\begin{tabular}{lll}
\hline Anti HCVNS3 expression & PMDs & OSCC \\
\hline Positive & $3(6 \%)$ & $7(7 \%)$ \\
Negative & $47(94 \%)$ & $93(93 \%)$ \\
Total & $50(100 \%)$ & $100(100 \%)$ \\
\hline
\end{tabular}

AntiHCVNS3, antibody hepatitis C virus non structural protein 3; OSCC, oral squamous cell carcinoma; PMDs, potentially malignant disorders

Table 2 Clinical parameters of different potentially malignant disorders (PMDs) included in the study

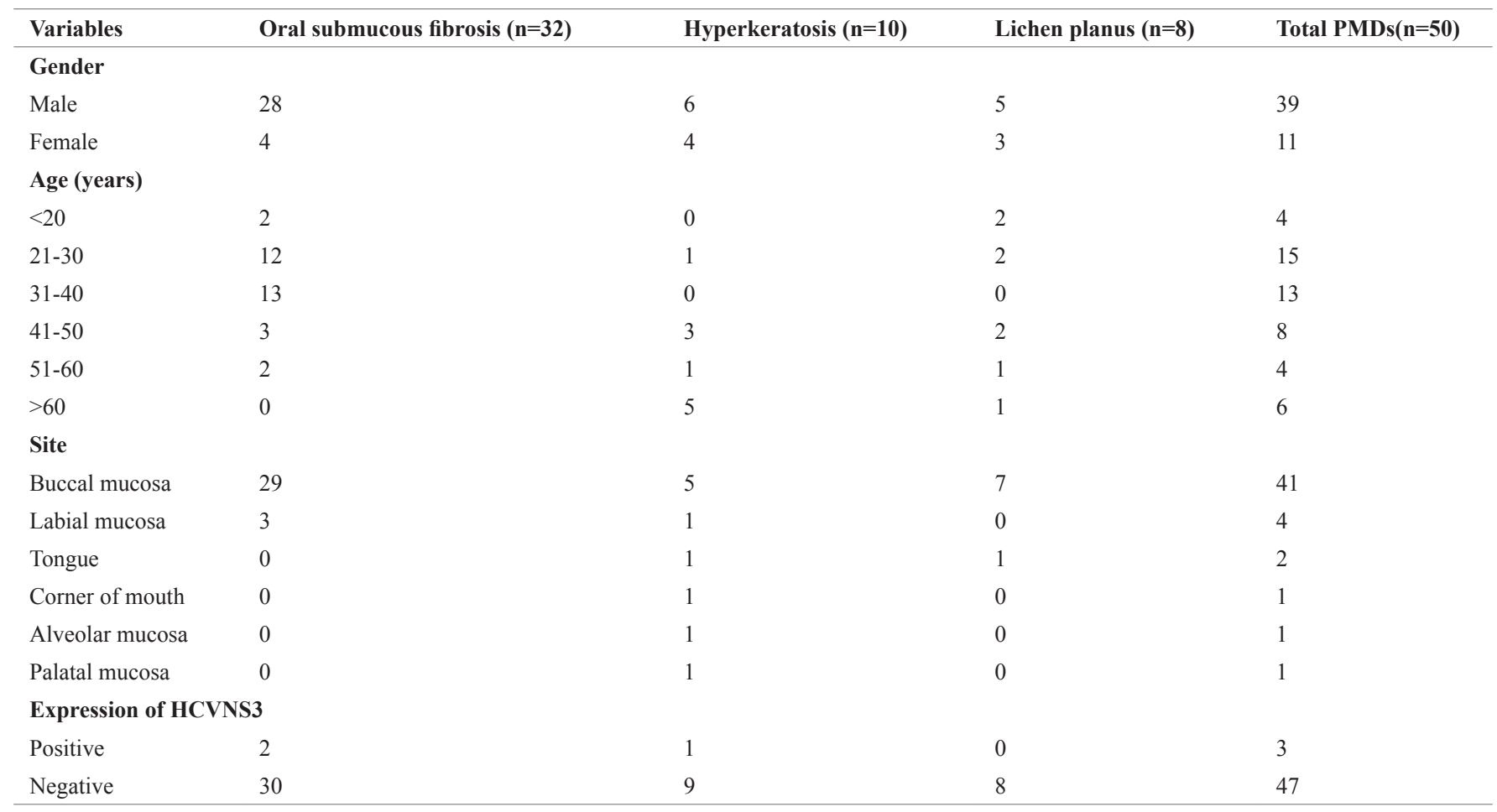

PMDs, potentially malignant disorders; HCVNS3, hepatitis C virus non structural protein 3 
Table 3 Clinical parameters of OSCC cases in association with HCV

\begin{tabular}{|c|c|c|c|}
\hline Variables & $\begin{array}{l}\text { HCV Positive OSCC } \\
(n=7)\end{array}$ & $\begin{array}{l}\text { HCV Negative OSCC } \\
(n=93)\end{array}$ & $\begin{array}{l}\text { Total OSCC } \\
(n=100)\end{array}$ \\
\hline \multicolumn{4}{|l|}{ Gender } \\
\hline Male & 6 & 70 & 76 \\
\hline Female & I & 23 & 24 \\
\hline \multicolumn{4}{|l|}{ Age (years) } \\
\hline$<20$ & 0 & I & 1 \\
\hline $21-30$ & 0 & 4 & 4 \\
\hline $31-40$ & 2 & 14 & 16 \\
\hline $4 \mid-50$ & 2 & 23 & 25 \\
\hline $51-60$ & 2 & 33 & 35 \\
\hline$>60$ & 1 & 18 & 19 \\
\hline \multicolumn{4}{|l|}{ Survival status } \\
\hline Alive & 0 & 49 & 49 \\
\hline Dead & 7 & 44 & 51 \\
\hline \multicolumn{4}{|l|}{ Bryne's grading } \\
\hline Good prognosis & 3 & 39 & 42 \\
\hline Moderate prognosis & 4 & 50 & 54 \\
\hline Poor prognosis & 0 & 4 & 4 \\
\hline \multicolumn{4}{|l|}{ Site } \\
\hline Buccal mucosa & 4 & 54 & 58 \\
\hline Gingivobuccal sulcus & 2 & 25 & 27 \\
\hline Palate & 0 & 5 & 5 \\
\hline Lip & 1 & 5 & 6 \\
\hline Tongue & 0 & 1 & 1 \\
\hline Floor of mouth & 0 & 3 & 3 \\
\hline
\end{tabular}

HCV, hepatitis C virus; OSCC, oral squamous cell carcinoma

Among 100 selected OSCC patients over the period of 5 years 49 patients were alive and 51 patients were dead. On the basis of Bryne's invasive cell grading 42 were of good prognosis, 54 of them were of moderate prognosis and 4 were of poor prognosis. On comparing all the three groups of Bryne's invasive cell grading with status of life, it was found that 26 out of 42,23 out of 54 and 0 out of 4 were alive in good prognosis, moderate prognosis and poor prognosis groups respectively. While, 16/42, 31/54 and 4/4 were dead in good, moderate and poor prognosis groups respectively.

The present study compared the expression of Anti HCVNS3 with different histological grades of oral squamous cell carcinoma by Bryne's invasive cell grading. On analysis, it was found that out of $7 \mathrm{HCV}$ positive OSCC, 3 were of good prognosis and 4 were of moderate prognosis. In this study we found that all the $7(100 \%) \mathrm{HCV}$ positive OSCC patients died within 2 years after surgical resection of the primary tumor. While, among $93 \mathrm{HCV}$ negative OSCC patients, 44 patients died among which $33(75 \%)$ died within a span of 2 years after surgery. This data contradicts the survival prediction with Bryne's invasive cell grading. As although HCV positive OSCC patients were of good and moderate prognosis they all died within 2 years of surgery suggesting there could be co-factors involved at molecular levels which contribute to poor survival of HCV positive OSCC patients. This data is unique to our study and it indicates that HCV positive OSCC patients could be a separate subtype of patients.

Hepatitis $\mathrm{C}$ virus is one of the major causes of chronic liver disease worldwide. A global estimated prevalence of HCV infection is $2.2 \%$, representing approximately 170 million infected people worldwide. The lowest prevalence of $\mathrm{HCV}(0.01 \%-0.1 \%)$ is in the United Kingdom and Scandinavia, whereas the highest prevalence is reported in Egypt (15\%-20\%) and intermediate rates (1.5\%-3.5\%) are found in United States, Japan, Spain and Italy. ${ }^{15}$ In India, the noted prevalence is below $2 \%$ and in Maharashtra $0.09 \%$. $^{16}$

Besides $\mathrm{HCV}$ is a potent risk factor for $\mathrm{HCC}$. An estimated $25 \%$ of hepatocellular carcinoma worldwide occurs in $\mathrm{HCV}$-infected patients. $^{17}$

In oral squamous cell carcinoma a diverse literature is present regarding co-infection with $\mathrm{HCV}$. It ranges from $21 \%{ }^{18}$ to $0.9 \%{ }^{19} \mathrm{No}$ study has been carried out in India till date. Also, extensive perusal of English speaking literature revealed the scarcity of data regarding the correlation of HCV with PMDs and OSCC. No immunohistochemical (IHC) study has been carried till date regarding this aspect. Hence, this 
study was carried out with the intention of addressing these scarcities in the literature with respect to $\mathrm{HCV}$ and its co-existence in PMDs and oral malignancies.

HCV-RNA is easily detectable in saliva from patients with chronic liver disease. ${ }^{20}$ This indicates that the cells in the oral region are continuously in contact to salivary or serum $\mathrm{HCV}$, which in turn increases the risk of genetic instability in the cells. ${ }^{21} \mathrm{HCV}$ is an RNA virus and it is thought to be unable to integrate its genome into the host genome. However, HCV proteins and the interaction between them and host proteins contribute to the viral oncogenic processes. ${ }^{22}$ Recently, host genetic factors including human leukocyte antigen (HLA) and cytokine genes have been implicated in HCV infection or persistence. ${ }^{23}$ Tumor-viruses induce oncogenesis through manipulating an array of different cellular pathways. These viruses initiate a series of cellular events, which lead to immortalization and proliferation of the infected cells by disrupting the mitotic checkpoint upon infection of the host cell. This is often accomplished by functional inhibition or proteasomal degradation of many tumor suppressor proteins by virally encoded gene products. The virally infected cells can either be eliminated via cell-mediated apoptosis or persist in a state of chronic infection. Importantly, the chronic persistence of infection by tumor viruses can lead to oncogenesis. ${ }^{24}$ The molecular pathways to carcinogenesis of $\mathrm{HCV}$ involve many signaling pathways such as MAP kinase, Wnt/ $\beta$-catenin, apoptosis, TGF- $\beta$, PI3K/Akt, NF$\mathrm{kB}$, Hedgehog, p53 and cytokine. All these signaling pathways are involved in cell proliferation, migration, invasion and metastasis. The activation of JNK signalling pathway is crucial for HCV nonstructural protein NS3-mediated cell growth. ${ }^{25}$

On comparing the expression of HCVNS3 in PMDs and OSCC we found that $6 \%$ and $7 \%$ PMDs and OSCC respectively were affected with $\mathrm{HCV}$. The almost equal incidence of $\mathrm{HCV}$ in PMD and OSCC could indicate 2 things:

$-\mathrm{HCV}$ infection is an early process in carcinogenesis.

-Thus, PMDs with HCV should be considered as high risk and should be kept under close observation for malignant transformation.

Limitations of the study: Simultaneous serum level comparisons should be done. But, this was not possible as it is a retrospective study. PCR confirmation could not be carried out.

Scope for further research: A large scale prospective study is warranted in Indian population to find the incidence of HCV in patients with oral squamous cell carcinoma. More detailed studies on association between $\mathrm{HCV}$ and potentially malignant disorders with follow up are warranted in the Indian population.

\section{Conclusion}

To conclude, we believe that HCV could be one of the factors out of multifactorial etiology involved in the development of OSCC. The coinfection with $\mathrm{HCV}$ in OSCC is indicative of poor survival in patients. These results warrant a multi-center analysis of incidence of $\mathrm{HCV}$ in PMDs and OSCC patients, and analysis of the molecular mechanisms involved in the pathogenesis of HCV in PMDs and OSCC.

\section{Acknowledgments}

I would like to thank Datta Meghe Institute of Medical Sciences for sponsoring the antibodies and providing infrastructural facilities to carry out this research. I would like to thank Mr. Rupesh Maladhari for all the technical help.

\section{Conflicts of interest}

Author declares no conflicts of interest.

\section{References}

1. Guha N, Boffetta P, Wünsch Filho V, et al. Oral health and risk of squamous cell carcinoma of the head and neck and esophagus: results of two multicentric case-control studies. Am J Epidemiol. 2007;166(10):11591173.

2. Sparano A, Quesnelle KM, Kumar MS, et al. Genome-wide profiling of oral squamous cell carcinoma by array-based comparative genomic hybridization. Laryngoscope. 2006;116(5):735-741.

3. Lin WJ, Jiang RS, Wu SH, et al. Smoking, alcohol, and betel quid and oral cancer: a prospective cohort study. J Oncol. 2011;525976.

4. de Martel C, Ferlay J, Franceschi S, et al. Global burden of cancers attributable to infections in 2008: a review and synthetic analysis. Lancet Oncol. 2012;13(6):607-615.

5. Alavian SM, Mahboobi N, Mahboobi N, et al. Oral conditions associated with hepatitis C virus infection. Saudi J Gastroenterol. 2013;19(6):245251.

6. Giordano TP, Henderson L, Landgren O, et al. Risk of non-Hodgkin lymphoma and lymphoproliferative precursor diseases in US veterans with hepatitis C virus. JAMA. 2007;297(18):2010-2017.

7. Nagao $\mathrm{Y}$, Sata M, Tanikawa K, et al. High prevalence of hepatitis C virus antibody and RNA in patients with oral cancer. J Oral Pathol Med. 1995;24(8):354-360.

8. Ghaderi R, Makhmalbaf Z. The relationship between Lichen Planus and Hepatitis C in Birjand, Iran. SEMJ. 2007;8(2):72-79.

9. Lodi G, Giuliani M, Majorana A, et al. Lichen planus and hepatitis $\mathrm{C}$ virus: a multicentre study of patients with oral lesions and a systematic review. Br J Dermatol. 2004;151(6):1172-1181.

10. Nagao Y, Tanaka J, Nakanishi T, et al. High incidence of extrahepatic manifestations in an HCV hyperendemic area. Hepatol Res. 2002;22(1):2736.

11. Nagao Y, Sata M,Kage M, etal. Histopathological and immunohistochemical study of oral lichen planus-associated HCV infection. Eur J Intern Med. 2000;11(5):277-282.

12. Grossmann Sde M, Teixeira R, de Aguiar MC, et al. Oral mucosal conditions in chronic hepatitis $\mathrm{C}$ Brazilian patients: a crosssectional study. J Public Health Dent. 2009;69(3):168-175.

13. Petti S, Rabiei M, Luca M, et al. The magnitude of the association between hepatitis $\mathrm{C}$ virus infection and oral lichen planus: meta-analysis and case control study. Odontology. 2011;99(2):168-178.

14. Jaber MA, Porter SR, Bain L, et al. Lack of association between hepatitis $\mathrm{C}$ virus and oral epithelial dysplasia in British patients. Int J Oral Maxillofac Surg. 2003;32(2):181-183.

15. Alter MJ. Epidemiology of hepatitis C virus infection. World $J$ Gastroenterol. 2007;13(17):2436-2441.

16. Mukhopadhya A. Hepatitis C in India. J Biosci. 2008;33(4):465-473.

17. Perz JF, Armstrong GL, Farrington LA, et al. The contributions of hepatitis $\mathrm{B}$ virus and hepatitis $\mathrm{C}$ virus infections to cirrhosis and primary liver cancer worldwide. $J$ Hepatol. 2006;45(4):529-538. 
18. Nobles J, Wold C, Fazekas-May M, et al. Prevalence and epidemiology of hepatitis $\mathrm{C}$ virus in patients with squamous cell carcinoma of the head and neck. Laryngoscope. 2004;114(12):2119-2122.

19. Eftekharian A, Khajavi M, Shokoofi S, et al. Hepatitis C virus in patients with squamous cell carcinoma of the head and neck in Iran: is there any relation? Eur Arch Otorhinolaryngol. 2012;269(12):2571-2573.

20. Komiyama K, Moro, Mastuda Y. HCV in saliva of chronic hepatitis patients having dental treatment. Lancet. 1991;338(8766):572-573.

21. Knudson AG. Hereditary cancer, oncogenes, and antioncogenes. Cancer Res. 1985;45(4):1437-1443.
22. Tsai WL, Chung RT. Viral hepatocarcinogenesis. Oncogene. 2010;29(16):2309-2324

23. Huang Y, Yang H, Borg BB, et al. A functional SNP of interferongamma gene is important for interferon-alpha-induced and spontaneous recovery from hepatitis C virus infection. Proc Natl Acad Sci USA . 2007;104(3):985990 .

24. Saha A, Kaul R, Murakami M, et al. Tumor viruses and cancer biology. Modulating signaling pathways for therapeutic intervention. Cancer Biol \& Ther. 2010;10(10):961-978.

25. Kanda T, Yokosuka O, Omata M. Hepatitis C Virus and Hepatocellular Carcinoma. Biology. 2013;2(1):304-316. 